\title{
Familiarity can increase stereotyping is
}

\author{
Eliot R. Smith ${ }^{\text {a,*, }}$, Daniel A. Miller ${ }^{\mathrm{a}, 1}$, Angela T. Maitner ${ }^{\mathrm{b}}$, Sara A. Crump ${ }^{\mathrm{b}}$, \\ Teresa Garcia-Marques ${ }^{c}$, Diane M. Mackie ${ }^{\mathrm{b}}$ \\ ${ }^{a}$ Purdue University, USA \\ ${ }^{\mathrm{b}}$ University of California, Santa Barbara, USA \\ ${ }^{c}$ ISPA-Lisbon, Portugal
}

Received 6 April 2005

Available online 19 September 2005

\begin{abstract}
Two experiments show that repeated exposure to information about a target person reduces individuation and thereby increases stereotyping of the target person based on social group memberships. The effect is not due to familiarity-induced liking (the mere exposure effect), nor is it mediated by increased accessibility of the target's social category, nor by increases in perceived social judgeability. The results are most consistent with the use of feelings of familiarity as a regulator of processing mode, such that familiar objects receive less systematic or analytic processing. In everyday life, frequent exposure to another person ordinarily produces not only familiarity but also liking, individuated knowledge, and friendship, factors that may effectively limit stereotyping. But when previous exposure is unconfounded from these other factors, its effect can be to increase stereotyping.

(C) 2005 Elsevier Inc. All rights reserved.
\end{abstract}

Keywords: Familiarity; Stereotyping; Person perception; Sytematic and heuristic processing

When people make decisions or judgments about other people, social groups, or other objects, sometimes they search with relative thoroughness for relevant information, combine the information appropriately, and arrive at a reasoned judgment. This type of systematic or thoughtful processing takes place when people possess both high ability (e.g., adequate information, freedom from distraction) and high motivation (e.g., due to the object's self-relevance). When ability or motiva-

\footnotetext{
Thanks to Sara McAllister, Michèle Banner, and Heather Claypool for research assistance. This work was supported by National Science Foundation grants BCS-0091807 and SBR-9209995, National Institute of Mental Health grants K02 MH01178 and R01 MH63762, and Fundação para a Ciência e Tecnologia Plurianual program ID332.

* Corresponding author. Present address: Department of Psychology, Indiana University, 1101 E. Tenth St., Bloomington, IN 474057007, USA.

E-mail address: esmith4@indiana.edu (E.R. Smith).

${ }^{1}$ Present address: Indiana University-Purdue University Fort Wayne, USA.
}

tion are lacking, people often engage in a much less effortful style of processing, based on simple cues or heuristics that allow them to assess an object or make a judgment with a minimum of time and thought. Dualprocess models elaborating these assumptions have been developed and applied in a number of different areas within social and cognitive psychology in the last couple of decades (Chaiken \& Trope, 1999; Sloman, 1996; Smith \& DeCoster, 2000).

Factors other than motivation and ability can also determine how people process information, and therefore the content of the judgments they ultimately make. One important factor is previous exposure to the target of processing. When people make judgments about objects that they have encountered previously, they tend to engage in lower levels of effortful, systematic processing (Garcia-Marques \& Mackie, 2000, 2001; Johnston \& Hawley, 1994). This tendency is adaptive because our processing capacity is limited and we encounter a myriad of objects each day. It would be wasteful for us to think 
extensively about objects, situations, or events that we have encountered in the past, because readily accessible knowledge should suffice to deal with these familiar objects. Instead, people should reserve extensive and thoughtful processing mostly for novel objects and situations.

Empirical evidence in several domains supports this hypothesis. Reder and Ritter (1992), studying problem solving, presented students with problems that were either novel or highly similar to previously-presented problems. If similar problems had been previously seen, the students tended to try to retrieve the answer (a relatively less effortful approach that draws on stored knowledge) rather than to effortfully compute an answer. In contrast, novel problems produced more effortful processing and less use of the retrieval-based strategy. Within social psychology, evidence supporting the same hypothesis comes from studies of participants processing persuasive messages. A persuasive message that participants have encountered previously is processed less analytically than the same message encountered for the first time (Claypool, Mackie, Garcia-Marques, McIntosh, \& Udall, 2004; Garcia-Marques \& Mackie, 2001; although see Cacioppo \& Petty, 1976).

In the area of person perception, the idea that previous exposure diminishes extensive analytic processing implies a strikingly counterintuitive prediction. In this domain effortful processes of individuation have often been contrasted with heuristic or non-analytic processes of stereotyping or category-based processing (Brewer, 1988; Fiske $\&$ Neuburg, 1990). There is considerable evidence that the "default" mode of person perception in the absence of either motivation or capacity is stereotyping, and that motivation and capacity tend to increase perceivers' use of individuating information (see Fiske, 1998). Intuitively, repeated exposure might be expected to increase the capacity to process information about the person and (perhaps) to increase motivation as well, allowing for greater individuation. Our prediction, however, is that previous exposure to information about a target person should decrease analytic processing of individuating information, thereby increasing the perceiver's reliance on stereotypes in making judgments about the target.

We hasten to add that one would not expect previous exposure to have this effect in many real-life relationships, because repeated encounters with a person should generally lead to increases in affective ties, depth of friendship, amount of individuated knowledge, and interpersonal interdependence. All these factors should, of course, motivate and enable individuated processing and thereby reduce stereotyping. However, in the laboratory, exposure can be experimentally unconfounded from these other factors. Equally important, "mere exposure" sometimes occurs in everyday life as well, because one might frequently see another person without engaging in meaningful interaction or forming an actual relationship. For example, one might frequently see someone who rides the same bus in the morning, works as a janitor in one's workplace, or (like a fast-food cashier) engages only in minimal, highly scripted interactions. In such cases, if repeated exposure indeed reduces analytic processing, we would expect increased stereotyping of familiar target persons, because this effect would not be counteracted by individuated knowledge, emotional involvement, and so forth.

Perceivers may obtain many different types of information about a target person (e.g., visual appearance, behaviors, verbal trait self-descriptions, etc.). What specific types of information, if repeated, might be predicted to lead to reduced analytic processing and hence increased stereotyping? Conceptually, the answer is that repetition of any part of the person information should cause this effect. Imagine, for example, a perceiver who obtains both visual-appearance and behavioral information about a target person. If either of these subsets of the information had been previously encountered, heuristic processing and greater stereotyping should result.

Note, though, that in studying this effect one must be careful to avoid a potential confound involving repeated exposure to cues for the stereotyped category itself. That is, a demonstration that repeated exposure to a target's visual appearance resulted in increased racial stereotyping would be of little interest. The stereotyping might not be due to familiarity alone, but due to repeated exposure to visual cues that indicate the person's racial category membership, making the category and its associated stereotype more accessible. A clear test of the hypothesis that repeated exposure per se increases stereotyping can be provided only if the repeatedly exposed subset of the information to be processed does not itself contain any cues to the stereotype, avoiding this potential confound.

This paper reports two experiments testing the hypothesis that repeated exposure increases the impact on social judgments of social group stereotypes, relative to individuating information. In Experiment 1, participants first briefly viewed photographs of a number of individuals, and then later saw some of those photos again, or comparable new photos, paired with information about the targets' occupations and behaviors. The photos (the repeated information) contained no cues to the targets' occupations. We expected that judgments about previously seen targets would be more consistent with occupational stereotypes, compared to judgments about novel targets.

\section{Experiment 1}

\section{Participants}

Participants were 44 introductory psychology students at Purdue University, who received research participation credit. 


\section{Procedure}

The experiment had two phases. In phase 1, participants viewed 30 photos of male and female faces on a computer monitor, each displayed for only $3 \mathrm{~s}$. Participants were instructed to try to remember the faces, which they were told would become relevant in an unspecified way later in the experimental session.

During phase 2, which occurred directly after phase 1 , participants were presented sequentially with 12 person descriptions. Each consisted of a photo of a face accompanied by a brief text description. Half of the photos were previously viewed by the participants in phase 1 (old) and half were new. Which photos were repeated and which were new were counterbalanced. The written descriptions included occupation labels and individuating information that was constructed to be somewhat inconsistent with the occupational stereotype. The occupational stereotypes used were accountant, librarian, artist, lawyer, professional boxer, hairdresser, stockbroker, waitress, truck driver, judge, mountain climber, and computer programmer. For each occupation, we chose three traits related to the occupational stereotype, based on informal pilot testing. For example, for the occupation of accountant, the stereotypic traits were boring, meticulous, and serious. We then constructed a brief person description that included somewhat counterstereotypical behaviors. Thus, the accountant description read: "I am an accountant. I live in Chicago. I love living in a big city because there is always something to do (ballgames, concerts, shows, etc.) Last week, I took off work to go to a Cubs game with a friend. At the ticket booth, my credit card was declined; I laughed, and asked my friend to cover it. It was definitely the best game I have ever seen."

\section{Dependent variables}

After reading each description, participants rated the person on five traits including two fillers and three traits related to the occupational stereotype. All trait ratings were made on 7-point scales. Greater stereotyping would be indicated by higher ratings on the stereotypic traits, whereas the use of individuating information would be indicated by lower ratings, since the behavioral information in each vignette tended to moderately (although not extremely) disconfirm the stereotype. We expected no effects on the filler trait ratings.

\section{Results and discussion}

A $2 \times 2 \times 2$ mixed ANOVA was conducted on the trait ratings, with the factors being old versus new photo (within subjects), counterbalance condition (between), and stereotype versus filler traits (within). We predicted an interaction of photo repetition with trait type, with the effect of old versus new photos being found only for the stereotypic traits and not for the fillers. The predicted interaction was significant, with $F(1,42)=5.11, p<.05$. To interpret the interaction, ANOVAs were run separately for the stereotypic and filler trait ratings. For the stereotypic traits, the effect of old versus new photos was significant with $F(1,42)=4.92, p<.05$. The mean rating on the stereotypic traits for old (previously seen) photos was 4.88 , and for new photos 4.71 . Thus, as predicted, the person descriptions accompanied by previously seen photos were rated more stereotypically than those accompanied by novel photos. In contrast, there was no effect of old versus new photos for the filler traits, $F(1,42)=0.44, p=.51$, means $=3.99$ and 4.05 .

Exposure is known to increase liking for familiar objects, in the classic "mere exposure" effect (Zajonc, 1968). Might the effect of exposure in this experiment be due to increased liking, rather than increased stereotyping? If so, the effect of exposure would be to make ratings higher for evaluatively positive stereotypes but lower for negative stereotypes. ${ }^{2}$ To test this possibility, we divided the stereotypes into those that were relatively positive versus negative, based on the valence of the dependent variable traits on which the stereotypes were rated. Adding valence as an additional within-subject factor in the analysis produced little change in the basic effect of previous exposure on the stereotype traits $(F(1,42)=4.78, p<.05)$. Valence had a strong effect $(F(1,42)=28.30, p<.0001)$, with the more negative stereotypes eliciting more stereotypical ratings than the positive ones. This valence effect may indicate that negative stereotypes had more weight in judgments overall than positive ones, although the effect is a function of the specific occupations and descriptions we used and cannot be unambiguously interpreted. Most important, valence did not interact with the exposure effect, $F(1,42)=1.83, p<.20$. Thus, the effect of old versus new photos was statistically consistent across the positive and negative stereotypes. Instead of previous exposure making ratings of old (compared to new) photos more positive, previous exposure made the ratings more stereotypic, on negative as well as positive stereotype-related traits. Thus, the results in this study are not due to the operation of the mere exposure effect.

Another potential alternative explanation is the suggestion by Mandler, Nakamura, and Van Zandt (1987) that familiarity might increase ratings on any type of judgment whatever, including the stereotype traits. However, this position is contradicted by more recent evidence that mere exposure leads consistently to

\footnotetext{
2 The main limiting condition on the increase of liking due to mere exposure, according to Bornstein's (1989) meta-analytic review, is boredom, which can be produced by large numbers of exposures to very simple stimuli. In this study, a single $3 \mathrm{~s}$ exposure to an interesting stimulus (a human face) should not lead to boredom or disliking.
} 
evaluative positivity and not to negativity (Bornstein, 1989; Reber, Schwarz, \& Winkielman, 2004). It is also inconsistent with our null effect of familiarity on the filler traits.

In summary, this experiment demonstrates that a brief previous exposure to a subset of information about a target person (a photo) can increase stereotyping of that target, even in the presence of counter-stereotypic individuating information. Exposure had a similar effect for both positive and negative stereotypes, effectively ruling out mere exposure-based positivity as a potential explanation of the results.

Experiment 2 was designed to further investigate our hypothesis that repeated exposure to targets makes them subjectively more familiar, which in turn signals that extensive processing is not necessary. We replicated the general design of Experiment 1, adding (for half the participants) a question about the subjective familiarity of each person description before the trait ratings. For participants who answer this question, the results should demonstrate that the previous exposure manipulation does produce a feeling of familiarity, as predicted. However, it has been demonstrated (Bornstein \& D'Agostino, 1992, 1994; Bornstein, 1989) that explicit awareness that a stimulus was previously encountered - an awareness that might be produced by answering the familiarity question - can undermine the effect of the previous exposure on other judgments (e.g., liking). Thus, responding to the question about familiarity might reduce or eliminate the effect of previous exposure on stereotypicality. However, for the participants who do not rate familiarity, we expect to replicate the result of Experiment 1.

\section{Experiment 2}

\section{Participants and design}

Fifty students from the University of California, Santa Barbara (21 men, 29 women) participated in this study for partial course credit. Participants responded to stimuli presented on computer screens using MediaLab software in individual cubicle spaces. Participants were randomly assigned to the cells of a 2 (repeated exposure: old versus novel face) $\times 2$ (perceived familiarity reported or not) design.

\section{Procedure}

As in Study 1, the experiment had 2 phases. In phase 1 , participants viewed 30 photos of male faces on a computer monitor (the face images used in this work were drawn from the CVL FACE DATABASE). Each photo was displayed for only $3 \mathrm{~s}$. Participants were instructed to try to remember the faces, which they were told would become relevant in an unspecified way later in the experimental session. Order of presentation was randomized across participants.

Next, the computer informed participants that they would begin the person perception task. In this phase, participants sequentially viewed each of six male faces paired with information about the target's occupation and some individuating information. Face-information pairings were counterbalanced across participants, and the targets were presented in a random order. Participants had viewed three of the target photos in the exposure phase (which targets had been exposed was counterbalanced across participants). Target descriptions were those of the librarian, lawyer, boxer, stock broker, truck driver, and accountant, described in Study 1.

\section{Dependent variables}

After viewing each face and reading the textual information, half of the participants were asked to rate how familiar each target was using a seven point scale $(1=$ "Not at all familiar," $7=$ "Very familiar"). All participants were then asked to make trait ratings (five traits for each target; three stereotypical of the target's occupation plus two fillers, neutral with respect to category membership) as well as a general likeability rating. The trait dependent variables were the same as in Experiment 1. Participants were finally debriefed, thanked, and dismissed.

\section{Results}

\section{Familiarity}

Participants asked to report target familiarity rated repeatedly exposed targets as more familiar $(M=3.50$, $S D=2.14)$ than non-repeatedly exposed targets $(M=2.50, S D=1.33) ; F(1,23)=85.09, p<.01$. Thus even the brief 3 -second previous exposure did make the repeated faces subjectively more familiar.

\section{Stereotyping}

Participants' ratings of the three stereotypic trait items for each target were averaged and subjected to a familiarity report $\mathrm{X}$ repeated exposure repeated-measures ANOVA. Analysis revealed a main effect of repeated exposure, $F(1,48)=8.26, p<.01$. Overall, repeated targets were rated more stereotypically $(M=3.00, S D=0.72)$ than novel targets $(M=2.70$, $S D=0.63)$. Although the two-way interaction was not significant, the effect of previous exposure differed somewhat between participants who did and those who did not answer the question about familiarity. Simple main effects tests showed that the difference between repeated $(M=3.11)$ and non-repeated targets $(M=2.72)$ was significant $(F(1,48)=7.31, p<.01)$ when participants were not asked to report familiarity, strongly replicating the results of Experiment 1. Participants who were asked to 
make familiarity ratings showed a weaker difference in the same direction (repeatedly exposed targets $M=2.88$, novel targets $M=2.68, F(1,48)=1.93, p<.18)$. Thus, when participants think explicitly about familiarity, they show a (non-significantly) smaller effect of repeated exposure in later judgments, directionally replicating an effect that has been demonstrated before (Bornstein \& D’Agostino, 1992, 1994).

Participants' ratings of the two filler trait items for each target were also averaged and subjected to familiarity report $\times$ repeated exposure ANOVAs. As in Experiment 1 , we found no differences between repeated exposure condition in trait ratings $(F(1,48)=1.02$, ns; overall $M=4.07$ ), nor did we find an effect of reporting familiarity or an interaction (both $F_{\mathrm{S}}<1$ ). Thus, participants' greater stereotyping of repeated targets again was not due to a general willingness to attribute any traits to those targets.

\section{Liking}

Neither the main effects of exposure or reporting familiarity, nor their interaction, had significant effects on liking, all $F \mathrm{~s}<1$. Thus this amount of repetition did not make targets more likable, further eliminating mere exposure as a possible mediator of the familiarity-stereotyping effect.

\section{Discussion}

First, this study demonstrated that repeated targets were indeed rated as more familiar than novel targets. This finding lends crucial support to our mediational hypothesis, that previous exposure produces feelings of familiarity, which in turn reduce analytic processing and thereby increase stereotyping. Second, the participants who were not asked to report familiarity replicated the results of Experiment 1: previous exposure to photos increased stereotyping. Thus, this study showed that the same previous exposure manipulation causes both increased familiarity and increased stereotyping.

Our hypothesis would be most definitively tested by a mediational analysis, using participants who reported familiarity to test whether rated familiarity mediates the effect of previous exposure on increased stereotyping. However, within that condition the effect of previous exposure on stereotyping was actually not significant, destroying the possibility of conducting such an analysis. This finding suggests that the act of reporting familiarity weakens the effect of previous exposure on judgments of stereotypicality. Other studies have shown, similarly, that cues that allow participants to attribute their feelings of familiarity to previous exposures of the stimulus show weaker effects of exposure on liking (Bornstein \& D'Agostino, 1992, 1994). In a similar vein, Bornstein's (1989) meta-analysis showed that subliminal exposures produce stronger mere exposure effects than supralimi- nal exposures, consistent with the idea that subliminal exposures do not allow this type of attribution and correction process. Our finding that reporting familiarity weakens the effects of the exposure manipulation on stereotypicality thus falls into line with a considerable body of prior research, and further implicates familiarity as the causal mechanism producing the effect.

The effect found in Experiment 2 is not due to a generalized willingness to attribute more of any trait to repeated targets, but as in Experiment 1 was specific to stereotypic traits. Finally, this effect was not due to mere exposure, or generally increased liking for repeated compared to novel targets.

This experiment also provides evidence against yet another possible alternative explanation, that prior exposure may increase "social judgeability" (Yzerbyt, Dardenne, \& Leyens, 1998). Social judgeability theory holds that when perceivers know only the social category of a target person, they may refrain from making stereotypic inferences because they do not feel entitled to make a judgment on that basis. Observe that our participants were not in the situation to which social judgeability theory is applicable, the situation of knowing only the target's social category. Participants received behavioral and visual appearance information as well as the social category (occupation). Nevertheless, one might propose a novel extension of social judgeability theory to argue that previous exposure may give perceivers the impression that they are more entitled to judge the person, freeing them to draw more heavily on the stereotype even though they also have individuating information available.

In this extended form, social judgeability is a possible alternative explanation for the results of Experiment 1, but not Experiment 2. This is because participants who had their attention explicitly called to the fact of previous exposure - those who rated the repeated targets as more familiar - would presumably feel the most entitled to make stereotypical judgments. However, participants who rated familiarity showed non-significantly less effect of previous exposure on the stereotypicality of their judgments, contradicting this extended version of the social judgeability hypothesis.

\section{General discussion}

These experiments were designed to test the hypothesis that previous exposure can increase stereotyping, by discouraging analytic processing. The size of the effect of previous exposure on stereotyping is similar in the two studies, with the effect size indices $d=.31$ and .44 respectively, conventionally described as small to medium size effects. The experiments provide evidence against three alternative hypotheses. Exposure can under some circumstances induce liking (through the classic mere 
exposure effect), but this effect does not match the pattern of our results in either of the experiments. In addition, previous exposure can lead to social judgeability and this might also encourage people to stereotype. However, we obtained evidence against this mediator in Experiment 2. Finally, Mandler et al. (1987) claimed that familiarity simply increased ratings on all traits, but neither of our studies showed increased ratings on irrelevant filler traits.

Why does previous exposure lead to stereotyping? As Garcia-Marques and Mackie (2001) and others (e.g., Johnston \& Hawley, 1994) have argued, this effect makes sense in terms of an organism's need to regulate processing effort. Since people's cognitive ability to process information in depth is limited (as assumed in all standard dual-process models; Smith \& DeCoster, 2000), we have to pick and choose situations and targets on which to focus extensive processing. Previous exposure is one reasonable and adaptive cue to use to regulate processing, because our previously gained knowledge should suffice to deal with previously encountered objects or situations. We can reserve the effort of carrying out extensive information-gathering and devising detailed plans of action for novel objects.

How does a perceiver know that an object has been previously encountered? The obvious answer is that previous exposure gives rise to a subjective feeling of familiarity. Several things can be said about the way familiarity arises as a function of previous exposure. First, familiarity can be induced by previous exposure to the whole body of information to be processed, or to salient sub-parts. For example, Garcia-Marques and Mackie (2001) exposed participants to a spoken persuasive message and then gave them a written version of the same message. In the studies in this paper, in contrast, participants were not repeatedly exposed to the entire person description that they ultimately judged, but only to a subset of it, the photo. Second, feelings of familiarity can result even when the presented information has not been encountered before in its exact form, but is similar to previously presented or previously known information. In the problem-solving study by Reder and Ritter (1992), for example, some problems were highly similar to previously-seen problems.

Third, the effect of familiarity should not depend on a conscious recognition by the perceiver that the information has been previously encountered, and a strategic decision to withhold processing effort. Much research including the seminal "false fame" studies of Jacoby and colleagues (Jacoby, Kelley, Brown, \& Jasechko, 1989) shows that previous exposure can lead to feelings of familiarity that can influence subsequent judgments, even when the person is unable to consciously recollect the prior exposure. Thus, feelings of familiarity can be dissociated from recollective judg- ments of previous occurrence (Jacoby \& Kelley, 1987; Mandler et al., 1987). Similarly, we suggest that vague feelings of familiarity should regulate processing even when people cannot consciously recognize that they have encountered the information before. Indeed, results of our Experiment 2 and Bornstein (1989) suggest that effects are even stronger when conscious recollection does not occur.

These studies demonstrate that when familiarity operates to regulate processing, people do not simply give the familiar information itself less processing. In our experiments the photo was familiar from prior exposure, while the text in each person description was novel. Still, the feeling of familiarity led participants to give more weight to the occupational stereotype (and less to the individuating behavioral information) even though the occupation was part of the written description, which was equally novel in all conditions. Our studies find, as the processing-regulation idea predicts, that the feeling of familiarity acts as a cue to generally reduce analytic processing of entire familiar objects - not just that the familiar subset of the information is given less analytic processing.

In making this suggestion, we advance what may appear to be a novel theoretical idea, that analytic processing is directed at specific stimulus objects, as in the familiar metaphor of the "attentional spotlight." This idea contrasts to the frequent interpretation that perceivers are "in a particular processing mode" (analytic or heuristic) at each point in time. We believe the latter cannot be the case. Analytic processing, given its limitedcapacity and sequential nature, can only be focused on a single object at a time. This means that even as one stimulus is being systematically considered, other perceptually available objects are not receiving the same type of intensive processing although they may still serve as heuristic cues. This point may be obscured because typical research designs only tap processing of a single stimulus (e.g., a persuasive message) at one time. However, we believe that it makes no theoretical sense to postulate that someone "in analytic processing mode" devotes equal processing effort to all available objects. Instead, when as in everyday life people are faced with a large array of stimuli, we believe that they use the items' relative familiarity to regulate processing, adaptively focusing more analytic processing on novel stimuli than on familiar ones.

The evidence reported in this paper leaves some open questions, of course. Although we were able to rule out several alternative hypotheses, we can provide no direct statistical evidence for familiarity as the mediator of increased stereotyping, because in Study 2 (as in some previous research) asking directly about familiarity weakened the effect itself. And a question remains about the breadth of the effect. Our theory states that familiarity tends to reduce analytic processing, thereby increas- 
ing the relative weight of stereotypes. But this logic presupposes that the stereotype itself can be readily noted without extensive processing. This assumption is consistent with the vast majority of the literature on stereotyping, which has focused on categories such as gender, race, or age that have immediate perceptual cues. But in a situation in which stereotype information is difficult to extract, we predict that familiarity would still lead to less analytic processing, but not necessarily to more stereotyping. This issue remains for future research.

How broad are the real-world implications of the fact that familiarity induced by previous exposure can lead to stereotyping? In most situations, of course, familiarity with another person is strongly correlated with individuated knowledge, affective bonds of friendship, etc.-factors that obviously tend to work against stereotyping even if a perceiver is processing non-analytically. However, when familiarity results from previous exposure that is unaccompanied by increases in these other factors (as in these studies), the resulting increase in stereotypical judgments can be observed. This type of unconfounding can occur in the real world as well as in the lab. Illustrating this possibility is a clever study of the mere exposure effect by Moreland and Beach (1992). The researchers arranged for several women to sit in on varying numbers of sessions of a large lecture course, where they sat quietly and took notes, although without interacting with the other students. At the end of the course, the other students were shown photos of the women and asked to make various ratings about them. The women who had attended the course more often-who were more familiar-were liked better and rated as more attractive, more intelligent, and generally more positive. The effect of familiarity on stereotyping, for which we have presented evidence in this paper, has additional implications. The women who were seen more often would likely have been rated as more emotional, more nurturant, more talkative, more dependent-in other words, in more female-stereotypical ways - on negative as well as positive aspects of the female stereotype. When commonplace situations such as seeing someone in a class numerous times over a semester can cause perceptions to become more stereotypic, it is a thought-provoking reminder of both the insidiousness and potency of stereotypes.

Broadening this idea, our results have another implication for real-world encounters with members of other groups. Psychologists long assumed that intergroup contact in the form of simple acquaintance would be sufficient to reduce prejudice (Amir, 1976). However, current thinking is that more intimate contact leading to true friendship is the key to reducing prejudice (Pettigrew, 1998). Our findings may suggest one reason for this fact: incidental contact, in the absence of more intimate famil- iarity, knowledge, and affective bonds, may actually tend to increase people's stereotyping of those they come in contact with.

\section{References}

Amir, Y. (1976). The role of intergroup contact in change of prejudice and race relations. In P. A. Katz (Ed.), Toward the elimination of racism (pp. 245-280). New York: Pergamon.

Bornstein, R. F. (1989). Exposure and affect: Overview and meta-analysis of research, 1968-1987. Psychological Bulletin, 106, 265-289.

Bornstein, R. F., \& D’Agostino, P. R. (1992). Stimulus recognition and the mere exposure effect. Journal of Personality and Social Psychology, 63, 545-552.

Bornstein, R. F., \& D’Agostino, P. R. (1994). The attribution and discounting of perceptual fluency: Preliminary tests of a perceptual fluency/attributional model of the mere exposure effect. Social Cognition, 12, 103-128.

Brewer, M. B. (1988). A dual process model of impression formation. In R. S. Wyer \& T. K. Srull (Eds.), Advances in social cognition (Vol. 1, pp. 1-44). Mahwah, NJ: Erlbaum.

Cacioppo, J. T., \& Petty, R. E. (1976). Effects of message repetition and position cognitive responses, recall, and persuasion. Journal of Personality and Social Psychology, 37, 97-109.

Chaiken, S., \& Trope, Y. (1999). Dual-process theories in social psychology. New York: Guilford.

Claypool, H. M., Mackie, D. M., Garcia-Marques, T., McIntosh, A., \& Udall, A. (2004). The effects of personal relevance and repetition on persuasive processing. Social Cognition, 22, 310-335.

CVL FACE DATABASE: http://www.lrv.fri.uni-li.si/facedb.html.

Fiske, S. T. (1998). Stereotyping, prejudice, and discrimination. In D. T. Gilbert, S. T. Fiske, \& G. Lindzey (Eds.), Handbook of social psychology (Vol. 1, fourth ed., pp. 357-414). Boston: McGraw-Hill.

Fiske, S., \& Neuburg, S. L. (1990). A continuum of impression formation form category-based to individuating processes: Influences of information and motivation on attention and interpretation. In L. Berkowitz (Ed.), Advances in experimental social psychology (Vol. 23, pp. 1-74). San Diego: Academic Press.

Garcia-Marques, T., \& Mackie, D. M. (2001). The feeling of familiarity as a regulator of persuasive processing. Social Cognition, 19(1), 934.

Garcia-Marques, T., \& Mackie, D. M. (2000). The positive feeling of familiarity: Mood as an information processing regulation mechanism. In J. Forgas \& H. Bless (Eds.), The message within: The role of subjective experiences in social cognition and behavior. Philadelphia: Psychological Press.

Jacoby, L. L., \& Kelley, C. M. (1987). Unconscious influences of memory for a prior event. Personality and Social Psychology Bulletin, 13, 314-336.

Jacoby, L. L., Kelley, C., Brown, J., \& Jasechko, J. (1989). Becoming famous overnight: Limits on the ability to avoid unconscious influences of the past. Journal of Personality and Social Psychology, 56, 326-338.

Johnston, W. A., \& Hawley, K. J. (1994). Perceptual inhibition of expected inputs: The key that opens closed minds. Psychonomic Bulletin and Review, 1, 56-72.

Mandler, G., Nakamura, Y., \& Van Zandt, B. J. (1987). Nonspecific effects of exposure on stimuli that cannot be recognized. Journal of Experimental Psychology: Learning, Memory, \& Cognition, 13(4), 646-648.

Moreland, R. L., \& Beach, S. R. (1992). Exposure effects in the classroom: The development of affinity among students. Journal of Experimental Social Psychology, 28(3), 255-276.

Pettigrew, T. F. (1998). Intergroup contact theory. Annual Review of Psychology, 49, 65-85. 
Reber, R., Schwarz, N., \& Winkielman, P. (2004). Processing fluency and aesthetic pleasure: Is beauty in the perceiver's processing experience? Personality and Social Psychology Review, 8, 364-382.

Reder, L. M., \& Ritter, F. E. (1992). What determines initial feelings of knowing. Familiarity with question terms not with the answer. Journal of Experimental Social Psychology: Learning, Memory and Cognition, 18, 435-451.

Sloman, S. A. (1996). The empirical case for two systems of reasoning. Psychological Bulletin, 119, 3-22.
Smith, E. R., \& DeCoster, J. (2000). Dual process models in social and cognitive psychology: Conceptual integration and links to underlying memory systems. Personality and Social Psychology Review, 4, 108-131.

Yzerbyt, V. Y., Dardenne, B., \& Leyens, J. P. (1998). Social judgeability concerns in impression formation. In V. Y. Yzerbyt \& G. Lories (Eds.), Metacognition: Cognitive and social dimensions (pp. 126156). Thousand Oaks, CA: Sage.

Zajonc, R. B. (1968). Attitudinal effects of mere exposure. Journal of Personality and Social Psychology, 9, 1-27. 